\title{
NEW GENERATION DIGITAL LONGITUDINAL FEEDBACK SYSTEM FOR DUKE FEL AND HI $\gamma$ S FACILITIES
}

\author{
Yujong Kim*, W. Wu, M. Busch, P. Wang, Y. K. Wu, DFELL, Duke University, NC 27708, USA \\ Dmitry Teytelman, Dimtel, Inc., Redwood City, CA 94061, USA \\ In-Soo Park and In Soo Ko, PAL, Pohang 790-784, Kyungbuk, Korea
}

\begin{abstract}
To cure harmful longitudinal coupled-bunch mode beam instabilities during high-current and multi-bunch beam operations at the Duke Free Electron Laser (FEL) and High Intensity $\gamma$-ray Source $(\mathrm{HI} \gamma \mathrm{S})$ facilities, we have been developing a new generation digital bunch-by-bunch longitudinal feedback (LFB) system which is based on an Integrated Gigasample Processor (iGp-64F). In this paper, we describe the new generation digital longitudinal feedback system for the Duke FEL and $\mathrm{HI} \gamma \mathrm{S}$ facilities.
\end{abstract}

\section{INTRODUCTION}

At the Duke FEL Laboratory (DFELL), there is a storage ring based OK-4/OK-5 FEL facility [1]. According to user applications, operating beam energy of the storage ring can be changeable from about $240 \mathrm{MeV}$ to $1.2 \mathrm{GeV}$. By changing beam energy of the storage ring and optical cavity mirrors, wavelength of coherent and ultra-bright FEL photon beams can be tunable from about $200 \mathrm{~nm}$ to about $800 \mathrm{~nm}$. The $\mathrm{HI} \gamma \mathrm{S}$ is generated by colliding those ultra-bright OK-4/OK-5 FEL photon beams with circulating electron beams through Compton back-scattering [1]. To generate the $\mathrm{HI} \gamma \mathrm{S}$ at a low photon beam energy of about $2 \mathrm{MeV}$ and to supply the spontaneous OK-4 wiggler radiation to the Photo-Emission Electron Microscopy (PEEM) users, operating beam energy of the storage ring should be around $280 \mathrm{MeV}$ [1], [2]. In that case, the natural damping is too slow to damp harmful coupled-bunch mode beam instabilities (CBMIs), which would be generated from time to time specially when stored beam current is high and beam energy is low [3]. To damp transverse CBMIs, an analog transverse feedback system was installed at the Duke storage ring in 2005. However, various strong longitudinal CBMIs were also generated during multi-bunch beam operation at a low beam energy [3]. According to our recent measurements with four symmetric filled buckets, the growth time of the strongest longitudinal CBMI is about $0.365 \mathrm{~ms}$ for a total beam current of $160 \mathrm{~mA}$ at $274 \mathrm{MeV}$, while the natural synchrotron radiation damping time is about $414 \mathrm{~ms}$. Therefore, to damp those longitudinal CBMIs, we have been developing an iGp-64F signal processor based longitudinal LFB system by collaborating with Dimtel, Inc. and Pohang Accelerator Laboratory (PAL). In this paper, we describe the new generation digital longitudinal feedback system for the Duke storage ring.

\footnotetext{
*yjkim@fel.duke.edu
}

06 Instrumentation, Controls, Feedback \& Operational Aspects
Table 1: Design parameters of the Duke LFB system.

\begin{tabular}{lcc}
\hline Parameters & Unit & Value \\
\hline beam energy & $\mathrm{MeV}$ & $240-1200$ \\
RF frequency $f_{R F}$ & $\mathrm{MHz}$ & 178.55 \\
revolution frequency $f_{0}$ & $\mathrm{MHz}$ & 2.78984375 \\
synchrotron frequency $f_{s}$ & $\mathrm{kHz}$ & $13.69-30.61$ \\
ratio of $f_{0} / f_{s}$ & $\cdot$ & $91-204$ \\
harmonic number &. & 64 \\
downsampling factor &. & $1-32$ \\
number of FIR taps & $\cdot$ & 16 \\
central frequency of kicker & $\mathrm{MHz}$ & $\sim 938$ \\
bandwidth of kicker & $\mathrm{MHz}$ & $\sim 90$ \\
shunt impedance of kicker & $\Omega$ & $\sim 1300$ \\
\hline
\end{tabular}

\section{NEW GENERATION LFB SYSTEM}

Generally, in the case of a time domain LFB system, no pre-knowledge on the dangerous coupled bunch modes is required while the pre-knowledge is required for a frequency domain LFB system [4]. Since we do not know exact source of impedance which generates strong CBMIs in the Duke storage ring, the time domain LFB system was selected to damp all harmful longitudinal CBMIs. Additionally, by saving bunch-by-bunch longitudinal phase motions at a memory, a digital LFB system can be usable to measure or monitor various useful beam diagnostic information such as bunch-by-bunch current, bunch-by-bunch synchrotron phase and its spread, growth and damping rates of the longitudinal CBMIs, and beam pseudo-spectrum which is the beam spectrum without revolution harmonics. After considering capability of arbitrary multi-bunch operation, developing time and cost, and advantages listed above, an iGp-64F signal processor based time domain digital LFB system instead of an analog one was selected for the Duke storage ring.

\section{Working Principles of New Digital LFB System}

The basic working principle of the new generation digital longitudinal feedback system is almost the same as that of the first generation [4]-[8]. Its design parameters are summarized in Table 1, and its detail layout and main components are shown in Fig. 1. The LFB system consists of three main components: a pickup BPM to detect the phase errors of circulating electron bunches, an iGp-64F signal processor to calculate feedback signals of those circulating bunches, a kicker to transfer those feedback signals to circulating electron bunches. Four signals of a pickup BPM

T05 Beam Feedback Systems 


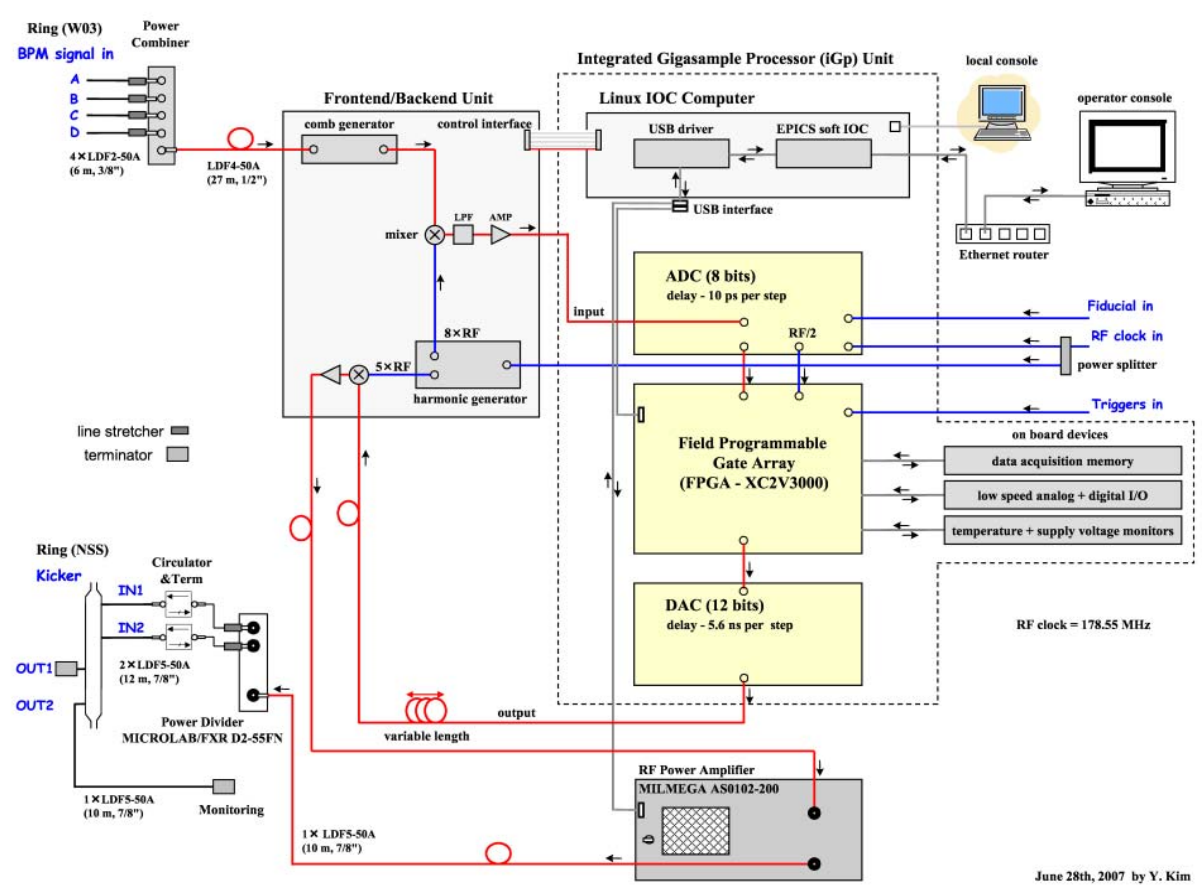

Figure 1: Layout of a digital longitudinal feedback system for Duke FEL facility.

are combined to remove dependence of those signals on transverse beam position. Then the BPM signal is transferred to a stripline comb generator to make a coherent tone burst at the eighth harmonic of the RF frequency. The phase error of circulating bunches is detected at a double balanced mixer where the signal from the comb generator is compared with one $1428.4 \mathrm{MHz}\left(=8 \times f_{R F}\right)$ signal coming from a harmonic generator. After sending the phase error signal to a low pass filter (LPF) to reduce the noise in the output of the double balanced mixer, the detected phase error signal is amplified by an amplifier. Then the phase error signal is digitized by an eight-bits analog-to-digital converter (ADC) at the bunch crossing rate of $5.6 \mathrm{nsec}$ for the full filled buckets.

Since the revolution frequency is much higher than the synchrotron frequency at the Duke storage ring as summarized in Table 1, all phase errors at every turn are not necessary to calculate a correction kicking output of the LFB system. By the help of the Nyquist sampling theorem, generally, four samples per one period are sufficient to reconstruct amplitude and phase of a sinusoid signal. However, the synchrotron oscillation or phase error oscillation is not a perfect sinusoidal signal. Therefore sixteen is selected as the number of taking phase error samples per one synchrotron period to restore synchrotron oscillations of circulating bunches, which is known as the downsampling [4], [5]. The downsampling is done after digitizing at the ADC.

The digital LFB system for the Duke storage ring has an iGp-64F signal processor which supports a baseband bunch-by-bunch processing channel for 64 bunches. According to beam energy or synchrotron frequency, the downsampling factor of the iGp-64F is adjustable from 1 to 32 , which supports any synchrotron frequency higher than $5.44 \mathrm{kHz}\left(=f_{0} / 32 / 16\right)$. Therefore the digital LFB system for the Duke storage ring can be operated at various beam energies from $240 \mathrm{MeV}$ with $f_{s}=30.61 \mathrm{kHz}$ to $1.2 \mathrm{GeV}$ with $f_{s}=13.69 \mathrm{kHz}$. After digitalization and downsampling of longitudinal phase errors, the feedback correction outputs are calculated by programmable 16-taps Finite Impulse Response (FIR) filter algorithm at a FieldProgrammable Gate Array (FPGA) [4], [5], [8].

Since those kicking outputs are independently calculated for all circulating bunches, all bunches have their own independent kicking outputs. Those kicking outputs are sent to the holder buffer before the 12-bits digital-to-analog converter (DAC). The holder buffer is a kind of memory where the most recent kicking outputs of all bunches are independently stored. Since phase errors are downsampled, those outputs are independently used to correct phase errors of circulating bunches until another new kicking outputs are calculated in a new synchrotron oscillation period by the iGp-64F signal processor. At the frontend/backend unit, the baseband kicking outputs are mixed with a kicker carrier signal whose a frequency is $892.75 \mathrm{MHz}\left(=5 \times f_{R F}\right)$. Since the central frequency of the Duke LFB kicker is about $937.3875 \mathrm{MHz}\left(=5.25 \times f_{R F}\right)$, the feedback gain is dropped by about $3 \mathrm{~dB}$ at higher frequency modes without the quad-phase-shift keyed (QPSK) modulator [4]-[7]. At the moment, the QPSK modulator for the new generation digital feedback system is not developed yet though it was used for the first generation digital feedback systems. Later, the frontend/backend unit for the new generation digital LFB system will be upgraded by including the QPSK modulator to avoid the gain dropping at higher 
frequency modes. After amplitude modulation, the kicking output signals are amplified by an RF amplifier. Finally, amplified kicking signals are transferred to circulating beams via the LFB kicker to compensate phase errors of electron bunches.

\section{Main Components of New Digital LFB System}

For the phase error detection, the frequency response of a pickup BPM should be flat for a wide frequency range. In our case, a spare arc BPM which had been installed in the west arc region was selected for this purpose. Its frequency response is good from DC up to about $4.0 \mathrm{GHz}$.

In the case of the iGp-64F signal processor unit, it had been developed by Dimtel, Inc. and already delivered to DFELL. The unit is much more compact and has much better performance than the digital signal processor farm for the first generation digital LFB system [4]-[8]. By upgrading the FPGA firmware, its maximum operating frequency can be increased up to $510 \mathrm{MHz}$ for 64 bunches. Since the iGp-64F signal processor unit has its own adjustable time delays at the ADC and the DAC, we do not need any additional time delays such as Colby delays. And in the same chassis of the signal processor unit, there is a Linux IOC computer with two USB interfaces where the USB driver and the EPICS IOC software are running. Therefore, no additional commercial real-time operating system such as VxWorks or Tornado is needed. The FPGA and a power amplifier are controlled by the IOC computer through those USB interfaces. For remote control and access, the IOC computer is connected to the overall control system via the Ethernet. In the case of the frontend/backend unit for the phase error detection and generation of a kicker carrier signal, it is under developing by Dimtel, Inc. now. It will be delivered to DFELL by the end of 2007.

In the case of the LFB kicker, DFELL has already finished physics and engineering design of a DA $\Phi$ NE type LFB kicker for the Duke storage ring by modifying the PLS LFB kicker [9], [10]. Its 3D geometry is shown in Fig. 2. Since its required bandwidth is about $90 \mathrm{MHz}\left(\simeq f_{R F} / 2\right)$ to damp all CBMIs, only two input/output ports are used. To consider a long bunch length of about 126.19 ps during FEL operation, $937.3875 \mathrm{MHz}$ was selected for its central frequency [3], [10]. Even its central frequency is somewhat lower than other LFB kickers, its shunt impedance was increased up to about $1300 \Omega$ by reducing bandwidth to $90 \mathrm{MHz}$, by adding a nosecone, and by optimizing geometry of the kicker [10]. Therefore an amplifier with about $200 \mathrm{~W}$ (rms) power is large enough to damp the fastest dipole mode CBMI within about $0.365 \mathrm{~ms}$ [3]. We purchased a $200 \mathrm{~W}$ amplifier from MILMEGA in 2007.

\section{SUMMARY AND FUTURE PLAN}

By collaborating Dimtel, Inc. and PAL, DFELL has been developing an iGp-64F signal processor based digital LFB 06 Instrumentation, Controls, Feedback \& Operational Aspects

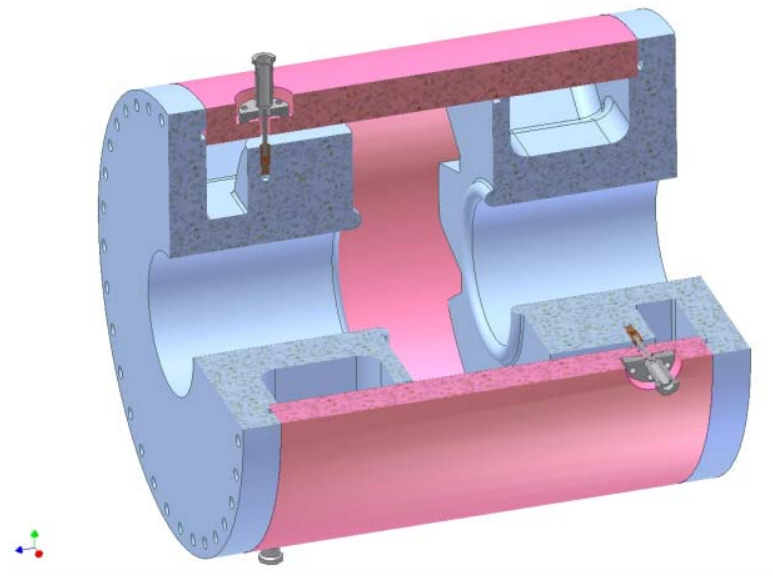

Figure 2: 3D geometry of the Duke LFB kicker.

system which is much more compact and has much better performance than its first generation. We expect that all harmful CBMIs which would be generated during PEEM and $\mathrm{HI} \gamma \mathrm{S}$ operations can be damped properly by installing the LFB system. All components of the LFB system will be installed by the end of 2007, and the commissioning of the LFB system will be started from January 2008.

\section{REFERENCES}

[1] V. N. Litvinenko, "Performance of High Intensity $\gamma$-Rays Source driven by the Duke Storage Ring FEL", in Proc. PAC2001, Chicago, USA, 2001.

[2] H. Ade et al., "A Free Electron Laser-Photoemission Electron Microscope System (FEL-PEEM)", Surface Review and Letters 5, 1257 (1998).

[3] Yujong Kim et al., "Growth Time of the Longitudinal Coupled Bunch Mode Beam Instability at Duke FEL Facility", in these proceedings.

[4] Yujong Kim et al., "Status of Longitudinal Feedback System for the PLS Storage Ring", in Proc. ICALEPCS1999, Trieste, Italy, 1999.

[5] G. Oxoby et al., "Bunch-by-Bunch Longitudinal Feedback System for PEP-II", in Proc. EPAC1994, London, U.K., 1994.

[6] J. Fox et al., "Programmable DSP-Based Multi-bunch Feedback - Operational Experience from Six Installations", SLAC-PUB-8410, 2000.

[7] Yujong Kim et al., "Commissioning Results of PLS Longitudinal Feedback System”, in Proc. EPAC2000, Vienna, Austria, 2000.

[8] D. Teytelman et al., "Design and Testing of Gproto Bunchby-Bunch Signal Processor", in Proc. EPAC2006, Edinburgh, Scotland, 2006.

[9] Yujong Kim et al., "Longitudinal Feedback System Kicker for the PLS Storage Ring”, IEEE Trans. Nucl. Sci. 47, 452 (2000).

[10] Wenzhong Wu et al., "Design of Longitudinal Feedback System Kicker for the Duke Storage Ring", to be published.

T05 Beam Feedback Systems 